Research Article

\title{
Application of Heart Rate Acceleration Motion Wireless Sensor Fusion in Individual Special Competitive Sports
}

\author{
Bu Chang (iD) \\ Sports Department, China Jiliang University, Hangzhou, China \\ Correspondence should be addressed to Bu Chang; 10b1502038@cjlu.edu.cn
}

Received 28 October 2021; Accepted 2 December 2021; Published 25 December 2021

Academic Editor: Gengxin Sun

Copyright ( 2021 Bu Chang. This is an open access article distributed under the Creative Commons Attribution License, which permits unrestricted use, distribution, and reproduction in any medium, provided the original work is properly cited.

\begin{abstract}
Heart rate monitoring is becoming more and more important in the development of modern health industry. At present, wireless sensor network equipment is mainly used to realize the real-time or periodic monitoring of human heart rate, so as to realize the health management of specific people. At the same time, the monitoring and analysis technology of heart rate is also widely used in special competitive sports. Through the real-time monitoring and analysis of athletes' heart rate, we can feedback and analyze their corresponding competitive state in real time, so as to monitor the sudden state of athletes, and also provide a basis for the improvement of athletes' later sports level. Based on this, this paper will use a single-chip microcomputer as the central data processing unit of the monitoring system at the hardware level, and inertial sensor and heart rate sensor at the sensor level. The system will design data acquisition module, motion positioning module, low-power module, athlete heart rate acquisition module, and motion state recognition module. Aiming at the low accuracy of traditional heart rate acceleration motion wireless sensor in competitive sports athletes' heart rate recognition and motion state recognition, this paper innovatively proposes an athlete heart rate recognition algorithm based on acceleration signal, which extracts the frequency-domain characteristics of motion signal. The time-domain and time-frequency characteristics of athletes' acceleration signal are used to realize the recognition of athletes' sports state, and the power spectrum cancellation technology is used to realize the accurate detection of athletes' heart rate. In order to verify the advantages of the hardware system and algorithm in this paper, three sports with quiet, dynamic, and random dynamic characteristics are selected for experimental verification. The experimental results show that the software algorithm proposed in this paper has obvious accuracy advantages in quiet and dynamic competitive sports compared with the traditional algorithm.
\end{abstract}

\section{Introduction}

The recognition, monitoring, and analysis technology of human heart rate and exercise state is widely used in health monitoring and exercise monitoring scenarios. At the same time, it is also an important index to evaluate exercise intensity and exercise quality. In professional competitive sports, real-time monitoring of human heart rate and corresponding sports status can not only fully obtain the energy consumed by athletes in the process of sports but also analyze their corresponding sports quality level. Systematic comprehensive analysis of the above two data can further improve athletes' sports level, improve defects in the process of sports, and improve sports quality [1-3]. The traditional heart rate detection of athletes is mainly based on hardware equipment such as heart rate belt, but the traditional hardware heart rate belt is inconvenient to wear, and its heart rate monitoring of athletes during exercise is not accurate [4]. The traditional athlete's movement state recognition technology is mainly embodied in three ways, which correspond to wearable sensor devices, external sensor devices, and wearable and hybrid integrated sensor devices, but these devices are more professional, and the corresponding equipment price and power consumption are relatively large, so they do not use athletes flexibly in competitive sports. It is convenient to wear, and the high price is not conducive to large-scale promotion [5-8]. Most of the detection algorithms of human heart rate and motion state also focus on the acceleration sensor algorithm, and a large number of heart rate and motion state recognition sensors are also 
developed based on this kind of algorithm. However, various algorithms lack relatively unified standards, so they are subject to various factors, and their corresponding heart rate monitoring accuracy and motion state recognition accuracy are not high. Therefore, based on the above discussion, it is of great significance and practical value to analyze and develop a heart rate acceleration detection device with low power consumption and high precision [9].

As an important technology in various application scenarios, wireless sensor technology is the product of the cross development of information technology and Internet of Things technology. Wireless sensor technology can realize the real-time monitoring of a specific scene environment or the corresponding state of a specific individual [10]. In traditional wireless sensor networks, a large number of wireless sensors are arranged at specific nodes to realize the collection, monitoring, and transmission analysis of specific data. Based on this, the main characteristics of traditional wireless sensor networks are as follows. One of their characteristics is distributed and self-organized. In this process, the corresponding nodes can be connected with each other through corresponding algorithms and through mutual monitoring and analysis, the corresponding data nodes have the ability of self-organization, and the corresponding network nodes will automatically configure and manage without affecting the operation of the sensor network. The second characteristic of wireless sensor network is that the corresponding sensor network data has large scale and high density, and the redundant information between corresponding nodes can realize cooperative work [11-13]. The third characteristic of wireless sensor networks is that the corresponding node energy of wireless sensor networks is relatively limited. The main energy consumption of conventional wireless sensor networks is mainly based on communication, which increases with the communication distance. Therefore, the main data transmission mode in many wireless sensor networks is multihop mode. This paper mainly uses wireless sensors. Inertial sensor and heart rate sensor are reasonably arranged based on these two sensors, and the corresponding collected information is timely summarized into the single-chip microcomputer for data analysis, and corresponding conclusions are given $[13,14]$. At the level of corresponding heart rate sensor, the main heart rate monitoring algorithms mainly include four calculation algorithms: blood oxygen heart rate monitoring, optical capacitance pulse wave heart rate monitoring algorithm, ECG algorithm, and arterial blood pressure method. These four traditional heart rate detection algorithms have various detection accuracy problems such as inaccurate detection in athletes' special competitive sports at the level of state recognition; the current inertial sensors mainly use acceleration sensors for motion state recognition. At present, such sensors have serious power consumption problems, so they do not meet the requirements of green low power consumption. At the same time, the relatively expensive price is not conducive to the wide promotion and use [15-18]. At the hardware system layout level, relevant scholars use the principle of hardware nearby layout to realize high-precision monitoring of key data, but this layout method will increase the vol- ume of the system and the corresponding system energy consumption will increase.

In view of the above corresponding research status and existing problems, this paper will analyze and verify the hardware system and software algorithm based on wireless heart rate acceleration sensor. Based on the above situation, this paper will use a single-chip microcomputer as the core processing unit of the system and design the data acquisition system with inertial sensor and heart rate sensor as sensing equipment module, motion positioning module and lowpower module, athlete heart rate acquisition module, and motion state recognition module. In view of the low accuracy of traditional heart rate acceleration wireless sensor in athletes' heart rate recognition and movement state recognition in competitive sports, this paper proposes an athlete's heart rate recognition algorithm based on acceleration signal to fully extract athletes' acceleration signal. At the same time, in the hardware design level, this paper optimizes the position of the sensor, so as to further optimize the hardware layout of the system and improve the detection accuracy of the sensor. In order to verify the advantages of hardware system and algorithm, three sports with quiet, dynamic, and random dynamic characteristics are selected for experimental verification. The experimental results show that compared with the traditional hardware and software, the hardware system and software algorithm have obvious accuracy advantages in quiet and dynamic competitive sports, but they have no obvious advantages in random competitive sports, which is also the focus of follow-up research in this paper.

The structure of this paper is arranged as follows: the second section of this paper will analyze and study the current research status of heart rate acceleration wireless sensor in competitive sports; the third section of this paper will carry out rational analysis and architecture design from the two aspects of hardware system and software algorithm of heart rate acceleration wireless sensor; the fourth section of this paper is mainly verification experiment and analysis; finally, this paper will give its summary.

\section{Correlation Analysis: Current Research Status of Heart Rate Acceleration Wireless Sensor in Competitive Sports}

At present, the application research of heart rate acceleration wireless sensor mainly focuses on health monitoring and sports condition detection of professional athletes. The main research contents include the design of hardware system and the research of software algorithm based on hardware system. Based on this, a large number of scientific research institutions and researchers have developed a variety of hardware systems and detection algorithms. At the hardware level, Japan and relevant European and American research institutions have developed a repeated athlete sports activity hardware system based on external sensors. It is mainly based on image sensors such as cameras and uses image sensors to recognize and detect the athlete's sports state. However, such hardware systems need a large number of 
storage devices to store the recognized image data. Therefore, the corresponding equipment cost is high [19]. Relevant universities in the USA have developed a five-axis human motion recognition and heart rate monitoring hardware system based on accelerometers. Based on this hardware sensing system, the corresponding heart rate monitoring accuracy and motion recognition accuracy are gradually improved, but the human body recognition accuracy in the moving state is low, and there are many sensors corresponding to the hardware system, so the cost of the hardware system is increased [20]. In order to improve the disadvantages of the five-axis sensor network, relevant European researchers have optimized and improved the five-axis sensor. The improved sensor is set as a three-axis sensor, and its corresponding three-axis accelerometer is mainly worn at the chest position of athletes. It realizes the static, transitional, and dynamic monitoring of the human body through the human chest characteristic signal and neural network monitor and analyzes the heart rate and movement [21]. Relevant Japanese scientists have fully combined the advantages of external sensors and wearable sensors to develop hybrid motion state and heart rate monitoring equipment. It mainly uses sensors and cameras to propose a general recognition algorithm, which mainly processes various characteristic information such as image, sound, heart rate, and posture of the monitored athletes and the corresponding detection of such equipment. The accuracy is higher than the other two, but the corresponding equipment cost is higher. At the same time, the corresponding algorithm is complex and the resource consumption is large [22, 23]. At the level of corresponding heart rate monitoring and motion state recognition algorithms, the mainstream algorithms include vector machine algorithm, naive Bayesian algorithm, decision tree algorithm, $k$-value proximity algorithm, and other algorithms; at the level of heart rate monitoring and analysis, relevant European scholars put forward heart rate monitoring algorithm based on pulse wave, which adds accelerometer sensors to athletes during exercise; the athletes' motion information is obtained separately, and the corresponding heart rate noise is removed by using the motion signal auxiliary algorithm. Finally, the transmission of heart rate signal is realized through Bluetooth and other wireless sensing technologies $[24,25]$. Based on the principle of photoelectric measurement, the corresponding researchers use multiple light-emitting diodes and photoelectric sensitive sensors to form the hardware part of heart rate monitoring. The photoelectric sensor detects the heart rate of athletes in competitive sports by sensing the change of light field intensity, but the accuracy of heart rate detected by this method still has errors [26-28].

\section{Application Analysis of Heart Rate Acceleration Motion Wireless Sensor Fusion in Individual Special Competitive Sports}

This section mainly analyzes the problems of heart rate monitoring and sports state recognition of athletes in com- petitive sports from the two aspects of hardware system and software system of heart rate acceleration motion wireless sensor. At the hardware system design level, the design is mainly based on a STM32 single-chip microcomputer. The design content includes the design of hardware module circuits such as data acquisition module, motion positioning module, low-power module, athlete heart rate acquisition module, and motion state recognition module. At the corresponding algorithm level, it mainly includes heart rate detection algorithm and motion recognition algorithm. This section mainly analyzes the principle and implementation process of the corresponding algorithm. The application principle block diagram of the corresponding heart rate acceleration motion wireless sensor in competitive sports is shown in Figure 1. From the figure, we can see the hardware and software logic block diagram of the system.

\subsection{Hardware System Design of Heart Rate Acceleration} Motion Wireless Sensor. At the hardware design level, the main application sensors of heart rate acceleration motion wireless sensor are inertial sensor and heart rate monitoring sensor. The corresponding core hardware includes CPU and its auxiliary circuit, data acquisition circuit, sensor circuit, wireless transmission circuit, positioning system module and its circuit, data storage part, and corresponding power management part circuit. The corresponding system hardware block diagram is shown in Figure 2. In some highspeed signal acquisition, this paper mainly depends on the related high-speed signal processing ad of ADI Company. At the same time, this paper optimizes the signal acquisition circuit on the PCB layout and fully considers the problem of signal integrity.

In the part of CPU and its auxiliary circuit design, the corresponding processor selection must have the characteristics of low price and low power consumption. Based on this, the processor selected in this paper is STM32. The processor has $\mathrm{m} 4$ core, which has rich resources, high cost performance, flexibility and stability, and relatively low power consumption. Based on this, the principle block diagram of the processor and its corresponding auxiliary circuit are shown in Figure 3. The corresponding data acquisition mainly collects the corresponding analog information through the ad module and transmits it to the central processing unit.

In the sensor hardware design part, it mainly includes inertial sensor and heart rate monitoring sensor. The inertial sensor mainly adopts a nine-axis inertial sensor, which integrates three-axis accelerometer, three-axis angular velocity, and three-axis magnetometer. At the same time, the sensor also has digital motion processor, so it has online programming processing function to a certain extent. Based on the sensor, the cost can be reduced, which mainly avoids the selection of complex peripherals and complex chips. The heart rate sensor used in this paper is mainly a biosensor based on pulse blood oxygen and heart rate function. Its specific composition includes LED lamp, infrared light, photoelectric detector, and subcircuit with environmental noise suppression ability. At the same time, the sensor hardware also integrates logic power supply and corresponding 


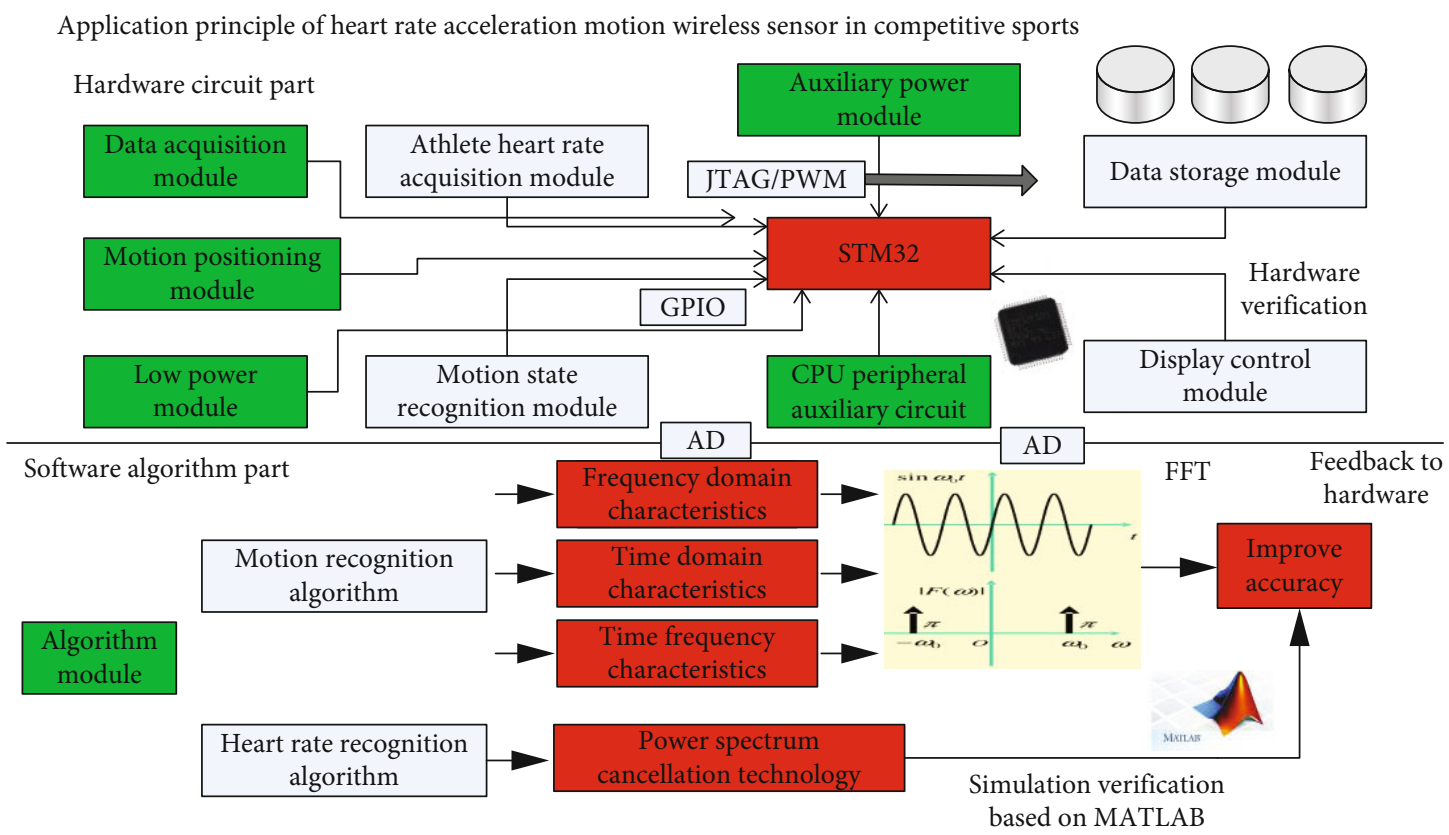

FIgURE 1: Application principle block diagram of heart rate acceleration motion wireless sensor in competitive sports.

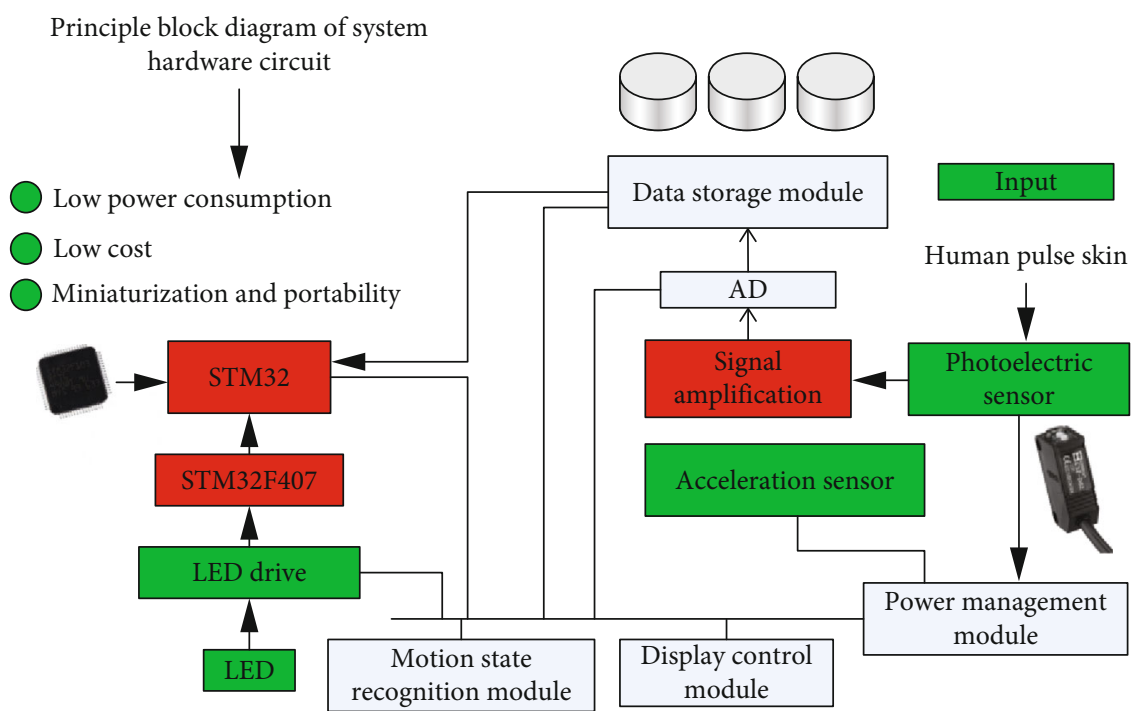

FIGURE 2: System hardware block diagram of heart rate acceleration motion wireless sensor in competitive sports.

subpower supply circuit. The heart rate sensor can transmit the collected data to the single-chip microcomputer through I2C interface, so as to detect and analyze the athlete's realtime heart rate through a certain data analysis algorithm. The physical sample corresponding to the sensor circuit module and the corresponding circuit principle block diagram are shown in Figure 4. From the figure, it can be seen that both the heart rate sensor and the inertial sensor have the characteristics of miniaturization and low power consumption.

In the corresponding wireless transmission and positioning part, the detection and positioning of athletes' sports state is mainly realized through GPS module, and the data transmission is realized through wireless transmission technologies such as Bluetooth or WiFi. The corresponding data storage circuit mainly realizes the basic reading and writing operation of data through SD card, which is also fully suitable for the application of a single-chip microcomputer system. C language is mainly used to read and write data storage. As an important power supply circuit of the system, the power management chip mainly selected in this paper includes mp1584 power conversion module and Texas Instruments related LDO chip. The corresponding wireless transmission module, CPU, and its auxiliary circuit are powered by LDO. The principle block diagram of the corresponding auxiliary power supply circuit and the 


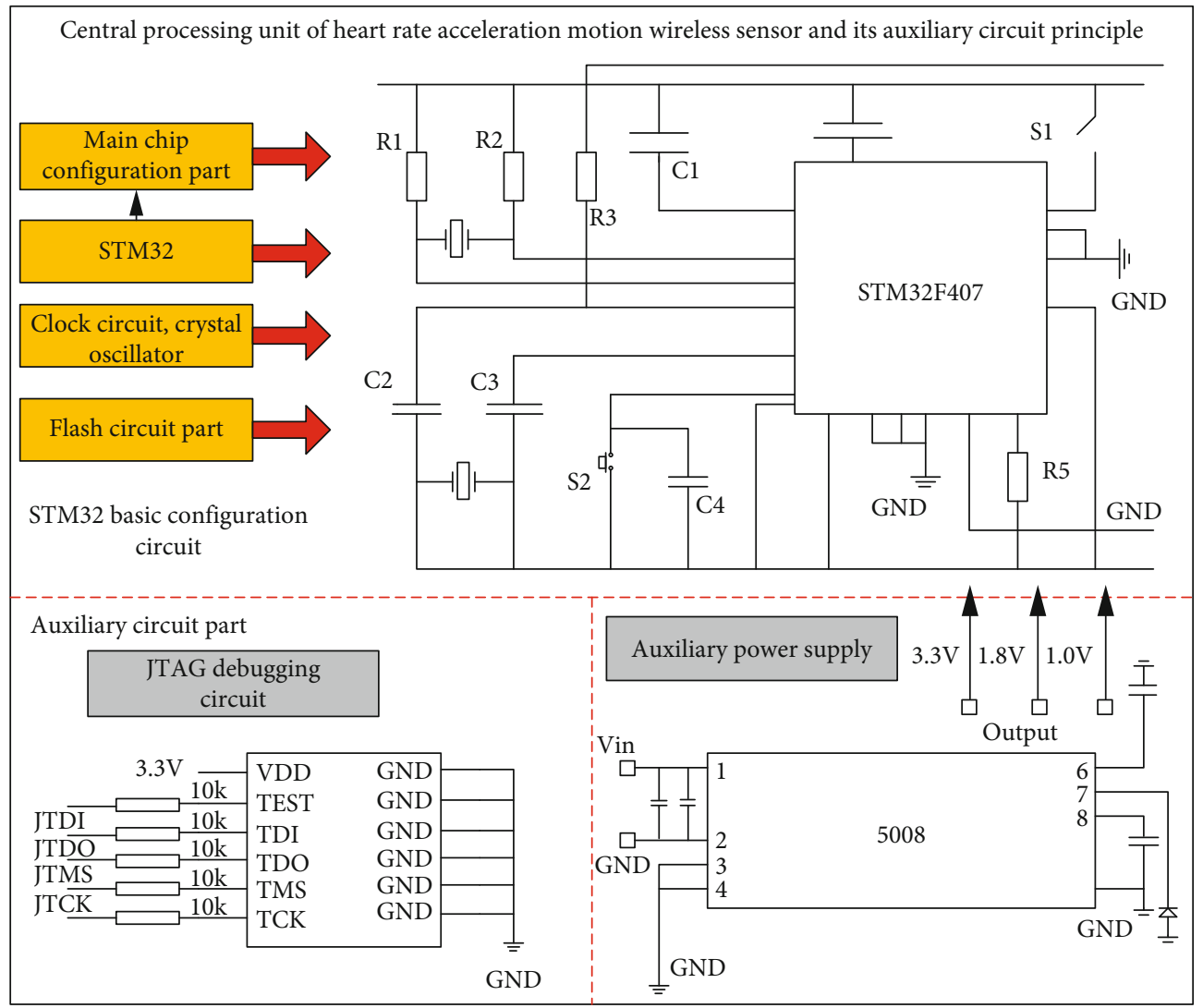

FIGURE 3: Heart rate acceleration motion wireless sensor CPU and its auxiliary circuit schematic diagram.

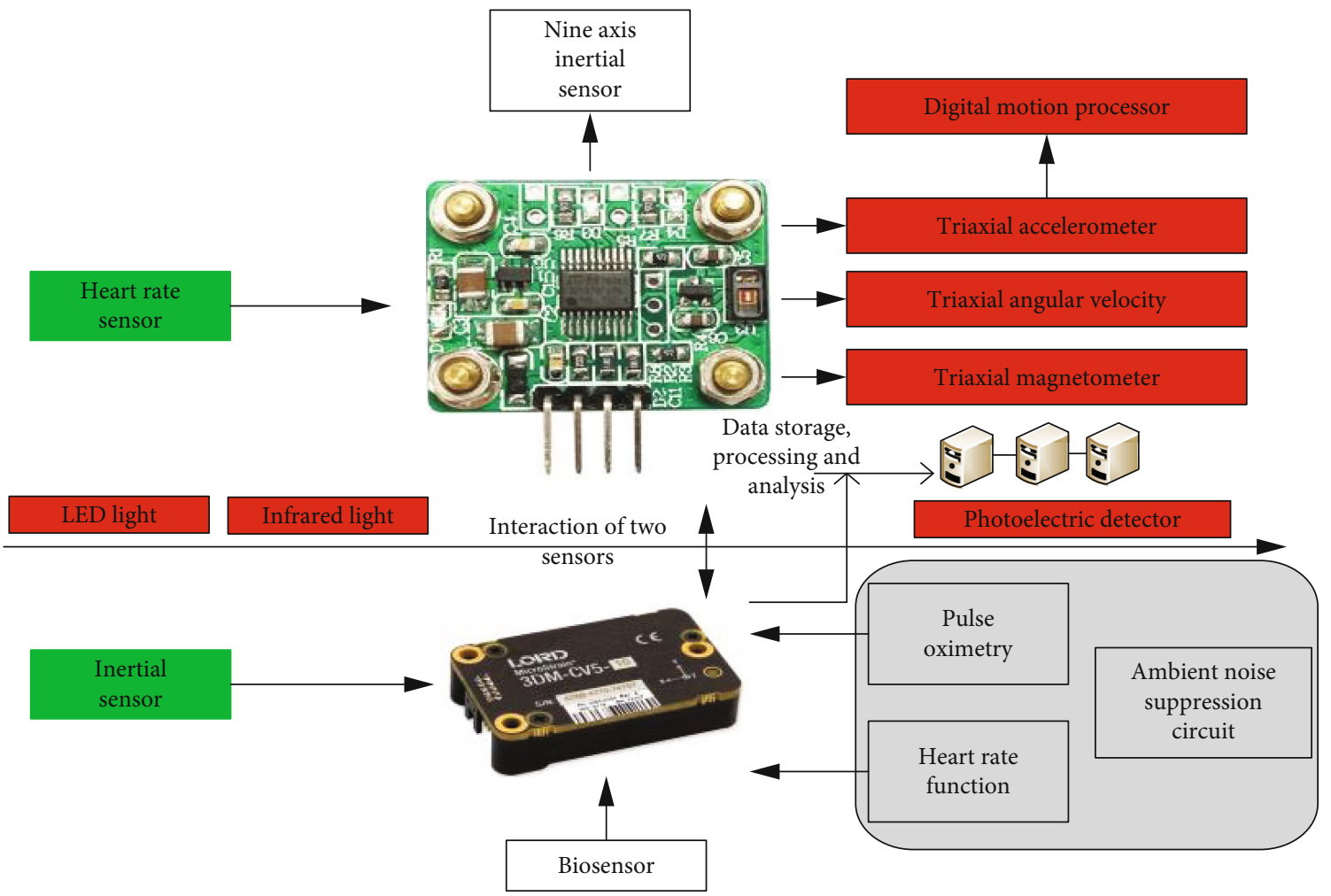

FIgURE 4: Circuit schematic diagram of sensor module in heart rate acceleration motion wireless sensor. 
corresponding physical diagram of the module are shown in Figure 5. From the figure, we can see the power supply demand and power supply relationship of each module in the system.

In the system low-power design level, this paper selects the low-power chip selected by each subsystem circuit module for the design and verification of the peripheral circuit. At the same time, this paper also takes into account the low cost of the chip when selecting the chip, so as to achieve the low-power and low-cost characteristics of the whole hardware system.

\subsection{Analysis of Heart Rate Acceleration Motion Detection} Algorithm Based on Wireless Sensor. In view of the low accuracy of traditional heart rate acceleration motion wireless sensor in athlete heart rate recognition and motion state recognition in competitive sports, this paper proposes an athlete heart rate recognition algorithm based on acceleration signal, which fully extracts the frequency-domain, timedomain, and time-frequency features of athlete acceleration signal to realize the recognition of motion state; the accurate detection of athletes' heart rate is realized by power spectrum cancellation technology.

Before optimizing the algorithm, this paper first studies and analyzes the interference factors of heart rate and analyzes the static, running, random exercise, and various stimulating exercises. The results show that the human heart rate is mainly subject to the interference and drift of human motion, which will produce a lot of burrs, drift, and unclear dominant frequency caused by irregular motion. Based on the above phenomena, this paper first preprocesses and analyzes the collected signals and filters them to a certain extent before entering the optimized heart rate monitoring algorithm. Carry out accurate heart rate analysis on the processed data. The principle block diagram of the corresponding optimized heart rate monitoring algorithm is shown in Figure 6. It can be seen from the figure that the interference of the external environment on the collected information can be further reduced by power spectrum weighting.

It can be further seen from Figure 6 that the principle of the corresponding optimized heart rate detection algorithm is as follows:

Step 1. Intercept a certain length of information fragment from the collected pulse information, perform fast Fourier transform on the information fragment, and perform mathematical operation as shown in Equation (1) on the Fourier transform corresponding to the pulse wave, so as to obtain a unified and standardized Fourier spectrum.

$$
\begin{aligned}
& X(i)=\frac{(X(1))^{2}}{\sum_{i=0,1, \cdots, n}^{i}(X(i))^{2}}, \\
& X(i)=\frac{(X(i))^{2}}{\sum_{i=0,1, \cdots, n}^{i}(X(i))^{2}} .
\end{aligned}
$$

Step 2. Considering the accuracy of the detected athlete's heart rate information, when selecting the data segment in Step 1 above, generally select a data segment of about $30 \mathrm{~s}$ $60 \mathrm{~s}$ and perform normalized Fourier transform for the data segment in this time period.

Step 3. Compare and analyze the Fourier transform of the processed pulse information with the motion Fourier spectrum and perform weighting operation. The corresponding weighting operation formula is shown in Equation (2). After the weighting operation, the corresponding motion components in the Fourier spectrum have been offset, and the spectrum information corresponding to the heart rate is highlighted.

$$
\begin{aligned}
& Y(i)=\frac{(Y(1))^{2}}{\sum_{i=0,1, \cdots, n}^{i}(Y(i))^{2}}, \\
& Y(i)=\frac{(Y(i))^{2}}{\sum_{i=0,1, \cdots, n}^{i}(Y(i))^{2}} .
\end{aligned}
$$

Step 4. Smooth the Fourier transform of motion signal, and the corresponding processing formula is shown in the following equations:

$$
\begin{aligned}
F(1)=\left[\frac{1}{2 * n}\right] *[F(1+m)], \\
F(i)=\left[\frac{1}{2 * n}\right] *\left[\sum_{m=-n}^{m=n-1}(F(i+m))\right], \\
\hat{F}(1)=X(1) * Y(1), \\
\hat{F}(i)=X(i) * Y(i) .
\end{aligned}
$$

Step 5. Use the heart rate data collected before and after the time period to weigh the secondary power spectrum and use the Gaussian function to construct the weighting function. The corresponding weighted pulse spectrum curve function is shown in the following equation:

$$
\begin{gathered}
\stackrel{\wedge}{P}(1)=X(1) * Y(1) * W(1), \\
\stackrel{\wedge}{P}(i)=X(i) * Y(i) * W(i) .
\end{gathered}
$$

Step 6. Select the peak point in the curve as the final athlete's heart rate value, record the heart rate value in each period of time, and construct the heart rate curve.

Based on the above steps, the accurate detection of athletes' heart rate in competitive sports can be realized, and the analysis of athletes' heart rate transformation process can be realized at the same time.

At the level of motion state recognition algorithm, this paper mainly realizes the recognition of motion state based on the frequency-domain characteristics, time-domain characteristics, and time-frequency characteristics of athletes' acceleration signal. In the corresponding frequency-domain feature extraction process, the main frequency parameters of 


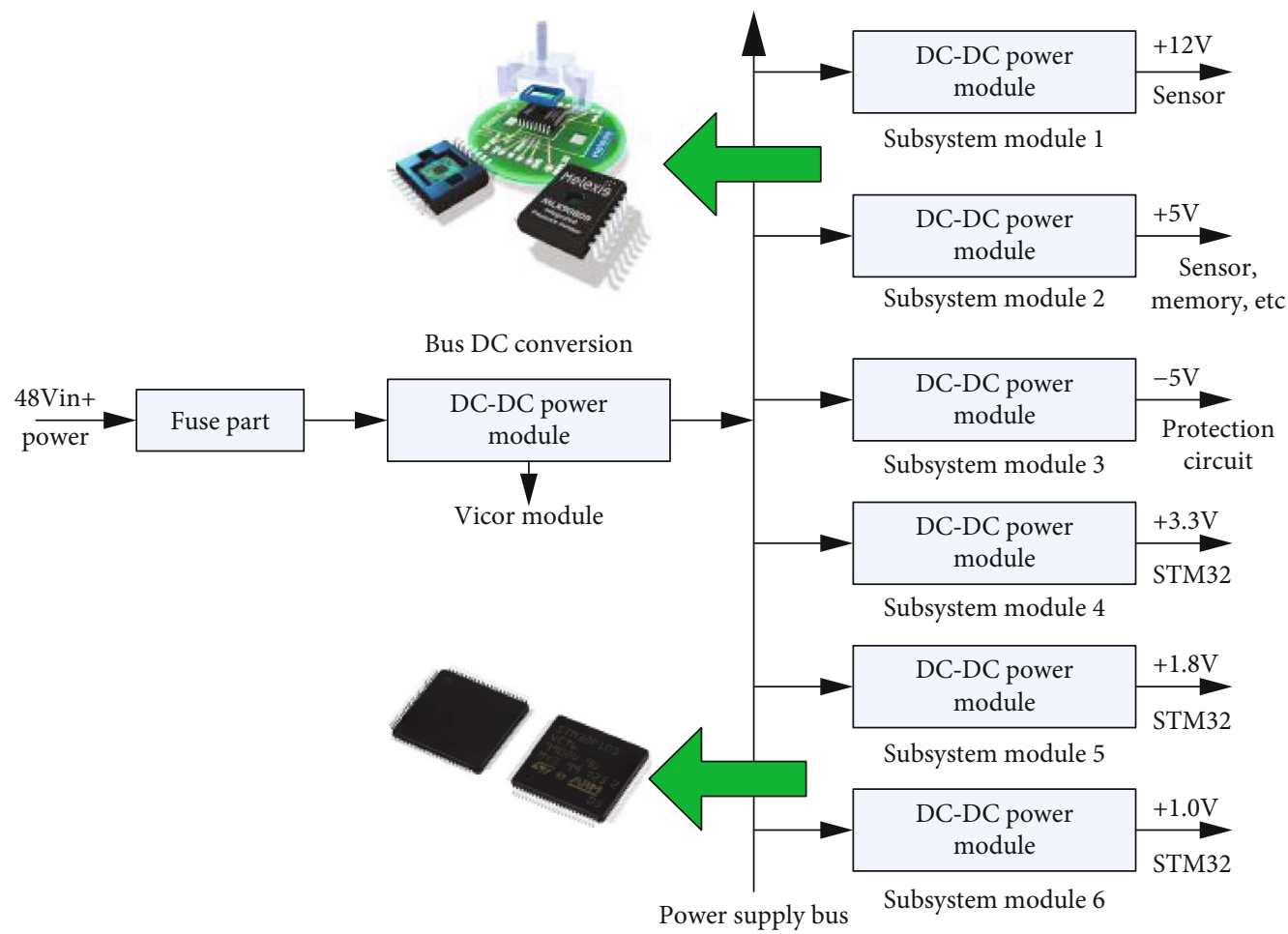

FIGURE 5: Circuit schematic diagram of power supply part in heart rate acceleration motion wireless sensor.

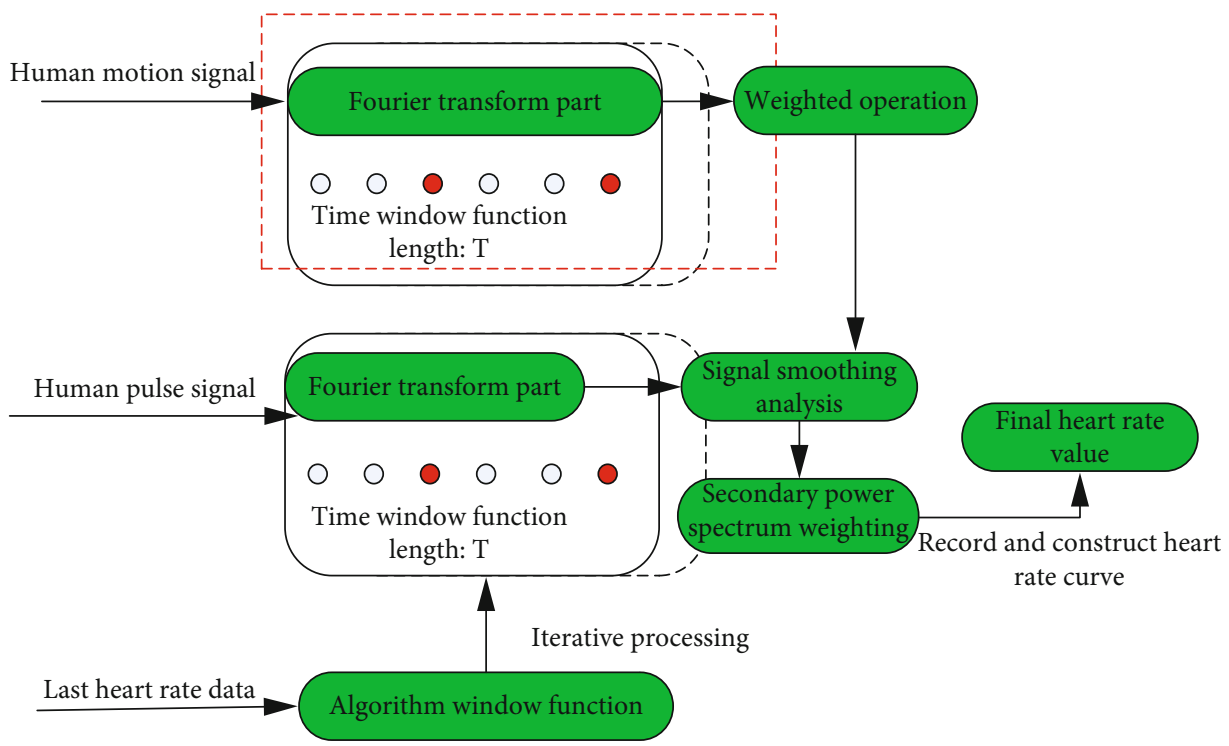

FIGURE 6: Principle block diagram of optimized heart rate monitoring algorithm.

motion information are extracted and taken as the main analysis object. The corresponding time-domain feature extraction mainly extracts the mainstream parameters such as the standard deviation of motion information and sample entropy. The extraction of corresponding time-frequency information is mainly based on the extraction and analysis of mixed parameters in time-domain and frequency-domain.

Based on the above two optimization algorithms and combined with the hardware design of the system, it can basically realize the accurate identification of athletes' sports state and heart rate information in competitive sports. At the same time, it has the characteristics of low power consumption, low cost, and high stability.

\section{Experimental Verification and Analysis}

In the experimental verification part, this paper mainly analyzes the athletes' movement state recognition and heart rate 

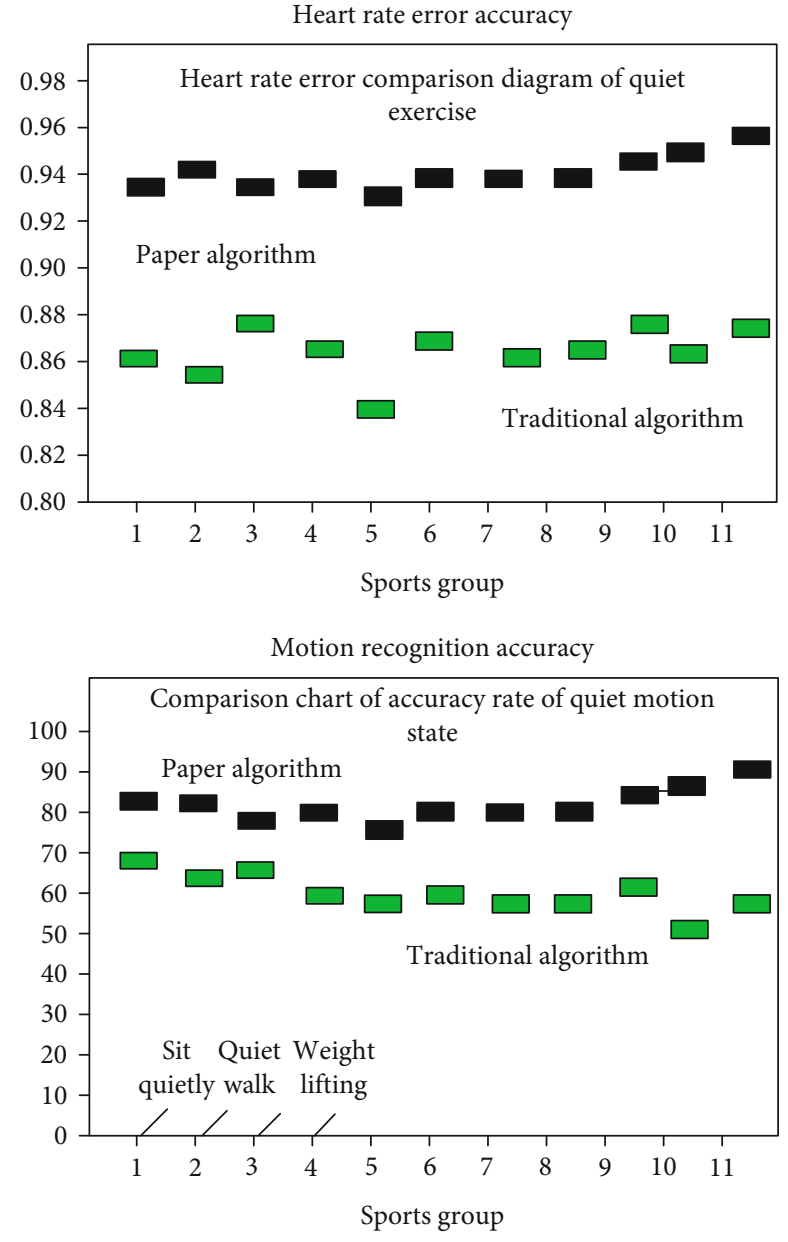

Figure 7: Comparison of heart rate error rate and exercise state accuracy under quiet exercise.

monitoring based on the above designed hardware system. The corresponding experimental conditions are as follows: 9 athletes were selected to perform static sports, random sports, and dynamic regular sports, and three athletes were assigned to each group. Based on the above three groups of athletes and the corresponding sports, the experiment is carried out and the experimental results are analyzed. The evaluation criteria of the experiment are mainly heart rate error rate index and motion recognition accuracy index. The above three experiments have different requirements for data acquisition, but their corresponding data acquisition circuits are basically similar, but the corresponding dynamic acquisition needs antishake processing. The corresponding description is shown in the highlighted part of the article.

Firstly, three athletes in quiet sports wear the hardware system designed in this paper, and based on this, collect the athletes' corresponding heart rate information and sports information. In the case of control variables, compare it with the traditional algorithm. The corresponding experimental results are shown in Figure 7; as can be seen from Figure 7, in quiet motion, the heart rate error of the algorithm proposed in this paper is obviously low, and the recognition accuracy of the corresponding motion information is high.
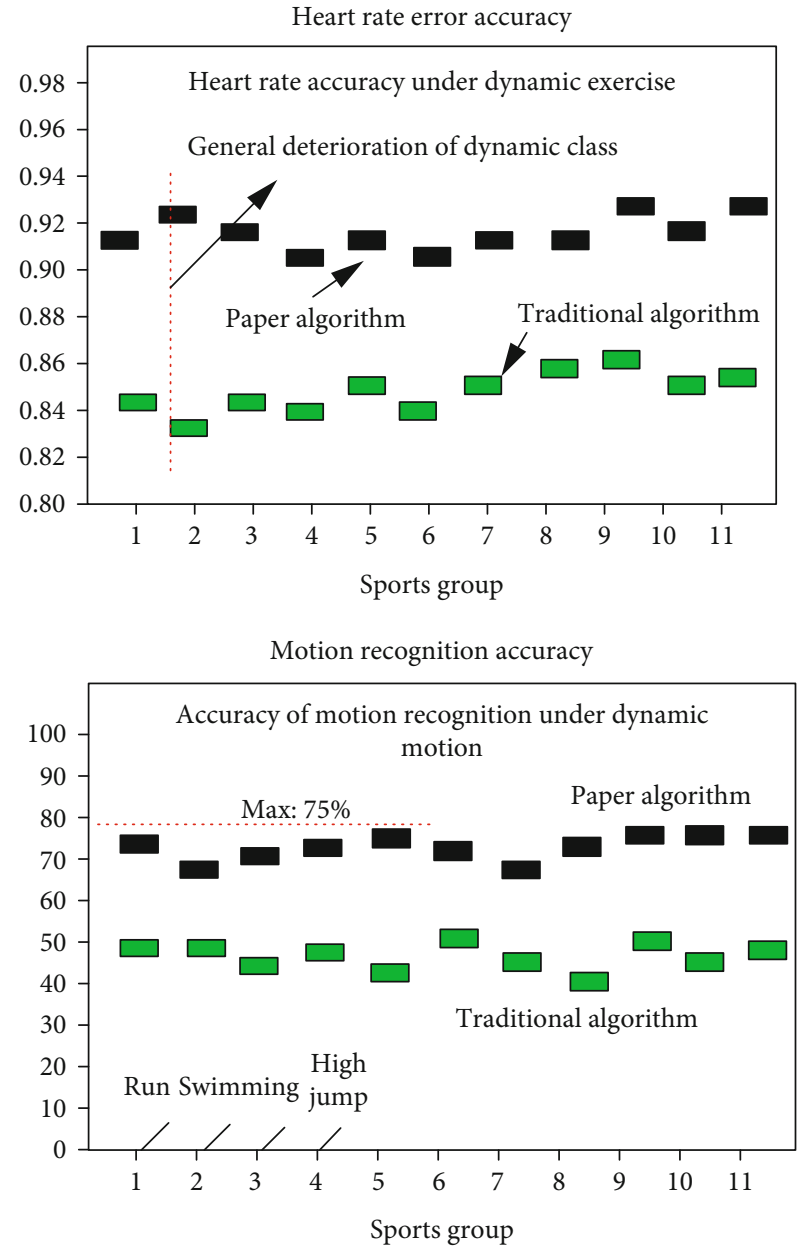

Figure 8: Comparison of heart rate error rate and exercise state accuracy under dynamic exercise.

For the corresponding athlete's heart rate information, heart rate error rate of sports information, and broken line diagram of sports state in the case of dynamic sports, as shown in Figure 8, it can be seen from Figure 8 that the heart rate error of the algorithm proposed in this paper is obviously low in dynamic sports, and the recognition accuracy of corresponding sports information is high. At this time, the corresponding heart rate error rate is relatively high compared with static motion, which also shows that the corresponding performance of this algorithm needs to be further improved when dealing with dynamic motion.

Heart rate information detection and motion state information monitoring experiments are carried out for random sports. The corresponding random sports in this paper mainly refer to basketball, because the dynamic and static properties of such sports are uncontrollable. The corresponding experimental results are shown in Figure 9. It can be seen from the figure that the algorithm proposed in this paper has detection accuracy and no longer has obvious advantages compared with the traditional algorithm. Therefore, it further points out the improvement of this algorithm in random motion. 

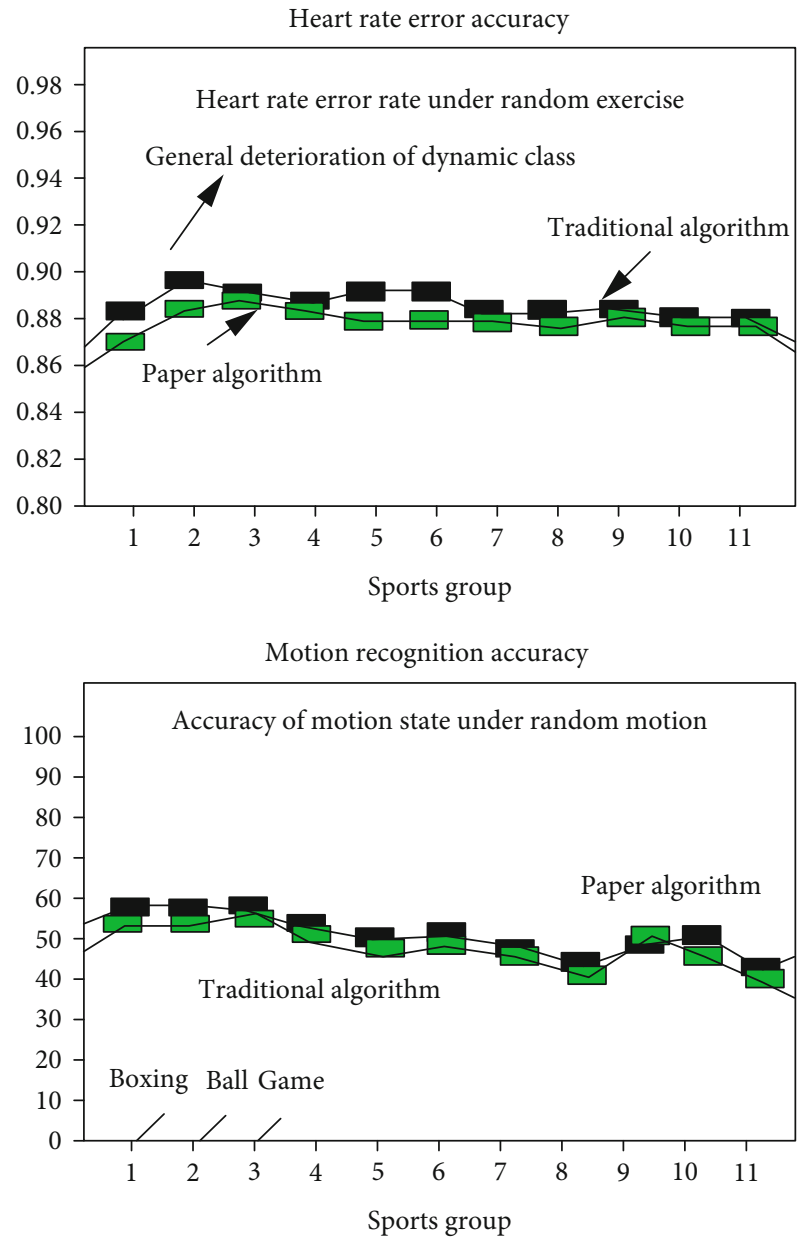

Figure 9: Comparison of heart rate error rate and exercise state accuracy under random exercise.

Power consumption (W)

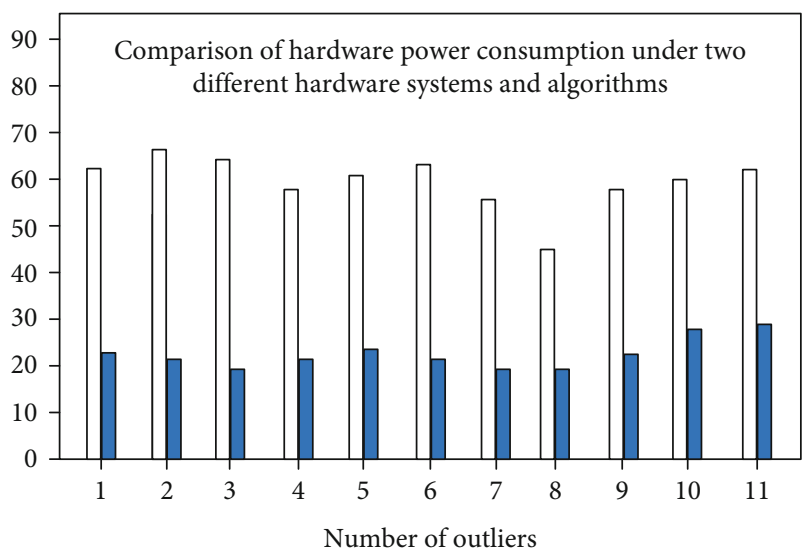

The power consumed by this algorithm

Power consumed by the algorithm

Figure 10: Comparison of hardware power consumption under two different hardware systems and algorithms.
In order to further verify the power consumption of the hardware designed in this paper, the power consumption comparison experiment is carried out with the traditional hardware system. When the control variables are guaranteed, the corresponding power consumption comparison line diagram of the corresponding two hardware systems under their respective algorithm consumption is shown in Figure 10. As can be seen from Figure 10, the hardware system and corresponding detection algorithm designed in this paper have lower power consumption under the same conditions, and their power consumption only accounts for about $70 \%$ of the traditional hardware system and its algorithm.

Based on the above experimental results and the corresponding experimental analysis, we can draw the following conclusions: compared with the traditional algorithms, the proposed algorithm has obvious detection accuracy advantages in quiet and dynamic motion, but its advantages are relatively small for random motion.

\section{Conclusion}

This paper mainly analyzes the research status of heart rate acceleration sensor fusion in individual special competitive sports and its disadvantages in hardware system components and detection algorithms. Aiming at the problem of low power consumption in the hardware system and the problem of low detection accuracy in the detection algorithm, this paper makes improvement and experimental verification. In the hardware part, this paper uses a single-chip microcomputer as the core processing unit of the system and uses inertial sensor and heart rate sensor as the sensing equipment. The system designs data acquisition module, motion positioning module, lowpower module, athlete heart rate acquisition module, and motion state recognition module. In view of the low accuracy of traditional heart rate acceleration motion wireless sensor in athlete heart rate recognition and motion state recognition in competitive sports, this paper proposes an athlete heart rate recognition algorithm based on acceleration signal, which fully extracts the frequency-domain, time-domain, and time-frequency features of athlete acceleration signal to realize the recognition of motion state; the accurate detection of athletes' heart rate is realized by power spectrum cancellation technology. In order to verify the advantages of the hardware system and algorithm in this paper, three sports events with quiet, dynamic, and random dynamic characteristics are selected for experimental verification. The experimental results show that the hardware system and software algorithm proposed in this paper have obvious accuracy advantages in competitive sports with quiet and dynamic characteristics compared with traditional hardware and software, but they do not have obvious advantages in competitive sports with random sports characteristics, which is also the focus of follow-up research in this paper. This paper will focus on the accuracy of athletes' data acquisition in the case of irregular movement. At the same time, this paper will 
continue to study the hardware optimization layout and signal integrity to make the system reach the best state.

\section{Data Availability}

The data used to support the findings of this study are available from the corresponding author upon request.

\section{Conflicts of Interest}

The author declares that there are no known competing financial interests or personal relationships that could have appeared to influence the work reported in this paper.

\section{References}

[1] N. Zhao, F. M. Li, and X. D. Liu, "Blind estimation of number of motion multi-human targets in wireless pyroelectric infrared sensor networks," Infrared Physics \& Technology, vol. 61, no. 1, pp. 208-215, 2013.

[2] S. Z. Yu, G. Zhu, and S. Niu, "Nanometer resolution selfpowered static and dynamic motion sensor based on micrograted triboelectrification," Advanced Materials, vol. 26, no. 11, pp. 1719-1724, 2018.

[3] H. Liu, Y. Wang, and K. Wang, "Turning a pyroelectric infrared motion sensor into a high-accuracy presence detector by using a narrow semi-transparent chopper," Applied Physics Letters, vol. 111, no. 24, article 243901, 2015.

[4] J. M. O'Kane and W. Xu, "Energy-efficient information routing in sensor networks for robotic target tracking," Wireless Networks, vol. 18, no. 6, pp. 713-733, 2012.

[5] B. H. Dobkin, "Wearable motion sensors to continuously measure real-world physical activities," Current Opinion in Neurology, vol. 26, no. 6, pp. 602-608, 2013.

[6] K. Martinez, J. K. Hart, P. J. Basford, G. M. Bragg, T. Ward, and D. S. Young, "A geophone wireless sensor network for investigating glacier stick-slip motion," Computers \& Geosciences, vol. 105, no. 3, pp. 103-112, 2017.

[7] R. Tu and L. Wang, "Real-time coseismic wave retrieving by integrated Kalman filter with observations of GPS, Glonass and strong-motion sensor," Advances in Space Research, vol. 53, no. 1, pp. 130-137, 2014.

[8] X. Zhong, M. K. Law, and C. Y. Tsui, "A fully dynamic multimode CMOS vision sensor with mixed-signal cooperative motion sensing and object segmentation for adaptive edge computing," IEEE Journal of Solid-State Circuits, vol. 121, no. 99, pp. 1-14, 2020.

[9] C. H. Wu and Y. C. Tseng, "Exploiting multi-spatial correlations of motion data in a body sensor network," IEEE Communications Letters, vol. 16, no. 5, pp. 662-665, 2012.

[10] B. Zhang, J. Chen, L. Jin et al., "Rotating-disk-based hybridized electromagnetic-triboelectric nanogenerator for sustainably powering wireless traffic volume sensors," ACS Nano, vol. 10, no. 6, pp. 6241-6247, 2016.

[11] Y. Sang, X. Huang, H. Liu, and P. Jin, "A vibration-based hybrid energy harvester for wireless sensor systems," IEEE Transactions on Magnetics, vol. 48, no. 11, pp. 4495-4498, 2012.

[12] B. Y. Li and P. J. Chuang, "Geographic energy-aware noninterfering multipath routing for multimedia transmission in wireless sensor networks," Information Sciences, vol. 249, no. 8, pp. 24-37, 2013.

[13] M. Hamed, I. Paul, and M. H. Craver, "An implantable wireless inductive sensor system designed to monitor prosthesis motion in total joint replacement surgery," IEEE Transactions on Biomedical Engineering, vol. 4, no. 9, pp. 11-21, 2019.

[14] T. H. Kim, S. H. Kang, D. S. Kim et al., "Feasibility study of patient motion monitoring by using tactile array sensors," Journal of the Korean Physical Society, vol. 67, no. 1, pp. 199-203, 2015.

[15] G. Dai, T. K. Aman, and F. Dimaio, “The HCN channel voltage sensor undergoes a large downward motion during hyperpolarization," Nature Structural \& Molecular Biology, vol. 26, no. 8, pp. 686-694, 2019.

[16] S. J. Paul, I. Elizabeth, and B. K. Gupta, "Ultrasensitive wearable strain sensors based on a VACNT/PDMS thin film for a wide range of human motion monitoring," ACS Applied Materials \& Interfaces, vol. 13, no. 7, pp. 8871-8879, 2021.

[17] C. Mzab, D. Zy, and D. By, "Making use of nanoenergy from human-nanogenerator and self-powered sensor enabled sustainable wireless IoT sensory systems," Nano Today, vol. 36, no. 4, article 101016, 2021.

[18] A. M. Silva, D. A. Santos, C. N. Matias et al., "Accuracy of a combined heart rate and motion sensor for assessing energy expenditure in free-living adults during a double-blind crossover caffeine trial using doubly labeled water as the reference method," European Journal of Clinical Nutrition, vol. 69, no. 1, pp. 20-27, 2015.

[19] J. S. Kim, Y. H. Jeong, and J. H. Park, “A geometric approach for forward kinematics analysis of a 3-SPS/S redundant motion manipulator with an extra sensor using conformal geometric algebra," Meccanica, vol. 51, no. 10, pp. 22892304, 2016.

[20] S. Alamandala, R. L. N. Sai Prasad, R. K. Pancharathi, V. D. R. Pavan, and P. Kishore, "Study on bridge weigh in motion (BWIM) system for measuring the vehicle parameters based on strain measurement using FBG sensors," Optical Fiber Technology, vol. 61, no. 3, article 102440, 2021.

[21] G. Peng, X. Liu, and S. Tang, "Enabling coverage-preserving scheduling in wireless sensor networks for structural health monitoring," IEEE Transactions on Computers, vol. 65, no. 8, pp. 2456-2469, 2016.

[22] N. Winer, W. D. Mason, C. H. Carter et al., "Effects of atenolol on blood pressure, heart rate, renin, and norepinephrine during exercise," Clinical Pharmacology \& Therapeutics, vol. 26, no. 3, pp. 315-325, 2016.

[23] D. Jarchi and A. J. Casson, "Towards photoplethysmographybased estimation of instantaneous heart rate during physical activity," IEEE Transactions on Biomedical Engineering, vol. 64, no. 9, pp. 2042-2053, 2017.

[24] C. R. Bellenger, J. T. Fuller, R. L. Thomson, K. Davison, E. Y. Robertson, and J. D. Buckley, "Monitoring athletic training status through autonomic heart rate regulation: a systematic review and meta-analysis," Sports Medicine, vol. 46, no. 10, pp. 1461-1486, 2016.

[25] G. Toni, M. Belvederi Murri, F. M. Piepoli et al., "Physical exercise for late-life depression: effects on heart rate variability," The American Journal of Geriatric Psychiatry, vol. 24, no. 11, pp. 989-997, 2016.

[26] D. Zammit-Mangion and M. Eshelby, "Evaluation of takeoff performance monitoring algorithm in large jet transport 
operations," Journal of Aircraft, vol. 43, no. 1, pp. 201-206, 2015.

[27] L. Cadmus-Bertram, R. Gangnon, E. J. Wirkus, K. M. ThraenBorowski, and J. Gorzelitz-Liebhauser, "The accuracy of heart rate monitoring by some wrist-worn activity trackers," Annals of Internal Medicine, vol. 166, no. 8, pp. 610-612, 2017.

[28] A. Yamamoto, H. Nakamoto, Y. Bessho et al., "Monitoring respiratory rates with a wearable system using a stretchable strain sensor during moderate exercise," Medical \& Biological Engineering \& Computing, vol. 57, no. 12, pp. 2741-2756, 2019. 